\title{
Reduce complexity by choosing your reactions
}

Mass spectra are built up by recording the abundance of ions at sequential mass-to-charge ratios $(\mathrm{m} / \mathrm{z})$ and can be used to identify what compounds are present in a sample. If a researcher wants to determine the presence or quantity of specific compounds, then the mass spectrometer can be programmed to look exclusively at their characteristic ions or fragmentation reactions. The development of selected ion monitoring (SIM) and, later, selected reaction monitoring (also called multiple reaction monitoring) allowed researchers to use such a strategy to achieve both greater sensitivity and quantitative accuracy.

Initially, SIM was performed using magnetic sector instruments. To do this, the magnetic field and the accelerating voltage from the ion source were adjusted to select up to three ions to monitor. A landmark paper from Bengt Samuelsson, Mats Hamberg and Charles Sweeley in 1970 showed that very sensitive and accurate quantitative analysis of nanogram amounts of the lipid vasodilator prostaglandin $\mathrm{E}_{1}$ could be achieved by adding a known amount of a deuterium-labeled version of the analyte (an internal standard) and then combining gas chromatography mass spectrometry (GC-MS; see Milestone 8) with SIM to measure the relative ion intensities of both the heavy and light versions. Building on this initial work, the use of isotopically labeled internal standards and SIM became routine for quantitative analysis in both GC-MS and liquid chromatography (LC)-MS.

Although SIM greatly reduced the complexity of GC-MS data, there were still many interfering compounds detected in the extracts of biological material; a further reduction in complexity was needed. In 1978, Richard Kondrat, Gary McClusky and R. Graham Cooks introduced selected reaction monitoring (SRM) by performing tandem mass spectrometry using a mass-analyzed kinetic energy spectrometer. They showed that, by monitoring a fragmentation reaction that was characteristic of a compound of interest, it was possible, for example, to detect cocaine in a sample of coca leaf without using any type of sample extraction or chromatography. The molecular ion for cocaine was selected using a magnetic sector and fragmented by introducing a collision gas through a second inlet. An electrostatic sector focused ions with the same kinetic energyin this case, the kinetic energy of a cation characteristic of cocaine fragmentation was chosen.

Today, the instrument of choice for SRM experiments is the triple quadrupole mass analyzer (Milestone 6). Richard Yost and Christie Enke were the first to demonstrate the advantages of this instrument for SRM, showing that up to 50 compounds or drug classes could be directly detected in a drug screen of blood extracts, and even from untreated serum. The analytical speed of the triple quadrupole meant that SRM could also be performed in real time for compounds eluting from GC and, later, LC columns.

SRM-especially in combination with standard isotope dilution-has found numerous applications in environmental, forensic and clinical analysis of small organic molecules. In modern triple quadrupoleinstruments, morethan 100 precursorproduct ion pairs can be recorded for

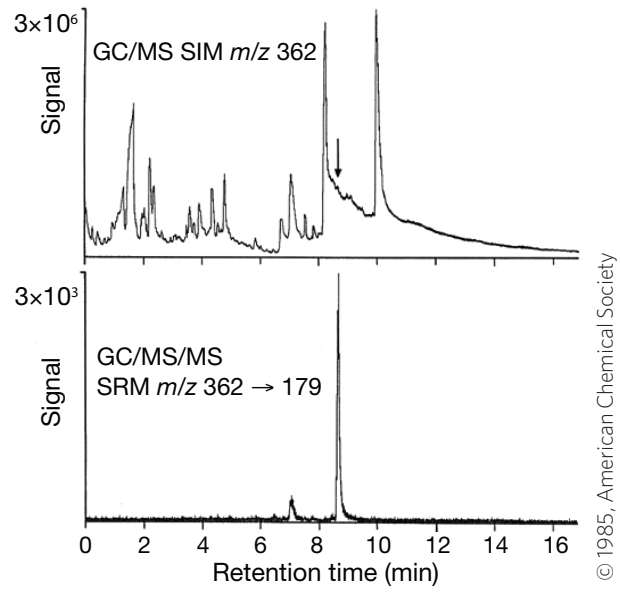

Targeted detection of methtryptoline in rat brain extracts. Adapted with permission from Johnson, J.V. \& Yost, R.A., Tandem mass spectrometry for trace analysis, Anal. Chem. $\mathbf{5 7}$ 758A-768A (1985).

peptides analyzed in an LC-MS run. Such advances have had a profound impact on the detection and quantitation of proteins, enabling a targeted proteomics approach akin to the use of antibodies for protein detection.

\section{Bronwen Dekker, Senior Editor,} Nature Protocols

ORIGINAL RESEARCH PAPERS Kondrat R.W., McLuckey, G.A.
\& Cooks, R.G. Multiple reaction monitoring in mass
spectrometry/mass spectrometry for direct analysis of complex
mixtures Anal. Chem. 50, 2017-2021 (1978) | Yost, R.A. \& Enke,
C.G. Triple quadrupole mass spectrometry for direct mixture
analysis and structural elucidation. Anal. Chem. 51, 231-243
(1979)
FURTHER READING Samuelsson, B., Hamberg, M. \& Sweeley,
C.C. Quantitative gas chromatography of prostaglandin E1 at the
nanogram level: use of deuterated carrier and multiple-ion
analyser. Anal. Biochem. $\mathbf{3 8}, 301-304$ (1970) | Brotherton, H.O. \&
Yost, R.A. Determination of drugs in blood serum by mass
spectrometry/mass spectrometry. Anal. Chem. 55, 549-553
(1983) | Gilette, M.A. \& Carr, S.A. Quantitative analysis of
peptides and proteins in biomedicine by targeted mass
spectrometry. Nat. Methods 10, 28-34 (2013)

